\title{
On the Optimality of Detectors Defined Over The Ambiguity Plane
}

\author{
Çag̃atay Candan \\ Middle East Technical University (METU) \\ Department of Electrical and Electronics Engineering \\ Ankara, Turkey. \\ ccandan@metu.edu.tr
}

\begin{abstract}
We study the detectors based on the ambiguity function. These detectors have been shown to be useful for the detection of linear frequency modulated (LFM) signals in the literature. We show that the detectors based on the projection of the cross-ambiguity surface are optimal for the detection of nonfluctuating targets in the Bayesian sense. The given optimality property strengthens the earlier results in the literature and enables us to compare different classes of detectors.
\end{abstract}

Index Terms-Detection, Neyman-Pearson Detectors, Bayesian Detectors, GLRT, LFM signals, Radar Receivers

\section{INTRODUCTION}

Radar systems are designed to reliably detect and accurately estimate the delay and Doppler frequency shift of the transmitted signal. The optimal Neyman-Pearson test for the presence or absence of a target at a hypothetical delay and Doppler shift values is the comparison of the cross ambiguity surface peak with a threshold. The optimality of this detector is valid for both slowly fluctuating targets under Rayleigh fading conditions [1, p.238] and non-fluctuating targets at low signal to noise ratio (SNR) conditions, [2, p.255].

In this paper, we use the following definition for the cross ambiguity function, [3, p.119]:

$$
\mathcal{A}_{r s}(\tau, u)=\int_{-\infty}^{\infty} r(t+\tau / 2) s^{*}(t-\tau / 2) e^{i 2 \pi u t} d t
$$

Here delay and Doppler frequency shift parameters are represented by $(\tau, u)$ respectively. The ambiguity surface is simply the magnitude square of ambiguity function, as defined in [4].

In many applications, some information on unknown target parameters, i.e. $(\tau, u)$, can be available before the detection. This can be the case for search-and-track radar systems or knowledge based systems with a-priori information on the spatial distribution clutter or interfering targets. For such systems, the optimal Bayesian detectors, which make use of given a-priori information, can be advantageous.

In this paper we examine detection methods over the ambiguity plane for two different types of detectors. The first type is the generalized likelihood ratio (GLRT) detector which treats $(\tau, u)$ as non-random but unknown parameters. The second type is the Bayesian detector which marginalizes the likelihood ratio of target-present and target-absent hypotheses over the joint probability distribution of $(\tau, u)$.
We show that the detector studied in [5] has some optimality properties under the Bayesian detection setting. The work presented in [5] is based on the projections of the ambiguity surface over the lines passing through the origin. This approach has been shown to be an effective parameter estimation solution for linear frequency modulated (LFM) signals.

There are some closely related studies in this line of research. Wang, Ozdemir and Akay independently propose to use Radon-ambiguity transform to detect chirp signals, [6], [7], [8], [9]. Among these Ozdemir et al. proposed a comprehensive detection method in [10] using projections over the ambiguity plane. The study presented here strengthens the earlier results in the literature.

Detection and parameter estimation of signals using timefrequency distributions has been studied extensively in the literature, [11], [12]. Flandrin studied detection over timefrequency plane in [13], [14]. The work of Flandrin has been instrumental for the development presented here. We use the work of Flandrin to show that the projections over ambiguity plane (which can be calculated through fractional Fourier transform) constitute the optimal detectors under Bayesian detection setting. Sayeed $e t$. al has also studied the optimal detection theory for bilinear time-frequency distributions in [15] (also see [16]). A valuable review article on timefrequency distributions with detection applications is [12].

The paper is organized as follows. In the next section we briefly review the connection between ambiguity surface and the fractional Fourier transform. This section is the summary of the work given in [5]. Next, we study the Bayesian and GLRT detection approaches and establish the connection between the Bayesian detector and detectors based on projections over ambiguity plane. The study concludes with comparisons and some remarks.

\section{Ambiguity FunCtion AND Fractional Fourier TRANSFORM}

The fractional Fourier transform generalizes the ordinary Fourier transform to a continuum of transforms. The definition of the fractional Fourier transform is given as

$f_{a}\left(t_{a}\right)=\mathcal{F}^{a}\{f\}\left(t_{a}\right)=A_{\phi} \int f(t) e^{i \pi\left(t_{a}^{2} \cot \phi-2 t_{a} t \csc \phi+t^{2} \cot \phi\right)} d t$ 
where $\phi=a \frac{\pi}{2}$ and $A_{\phi}$ is a unit norm constant depending on $\phi$. When $a=1$, the definition in (2) becomes identical to the definition of the ordinary Fourier transform. When $a \rightarrow 0$, the transform approaches identity transform, that is as $a \rightarrow 0$, $f_{a}\left(t_{a}\right) \rightarrow f(t)$ and the kernel of the transform approaches $\delta\left(t-t_{a}\right)$. For any value of $a, f_{a}\left(t_{a}\right)$ is a unitary transform of $f(t)$. Readers can consult [17] for more information on the transform and its applications.

We briefly examine the connection between fractional Fourier transform and the ambiguity function. The following relations are well known for the ambiguity function:

$$
\begin{aligned}
A_{r, s}(\tau, 0) & =\int_{-\infty}^{\infty} r(t) s^{*}(t-\tau) d t \\
\int_{-\infty}^{\infty}\left|\mathcal{A}_{r, s}(\tau, u)\right|^{2} d u & =\int_{-\infty}^{\infty}|r(t)|^{2}|s(t-\tau)|^{2} d t
\end{aligned}
$$

The relation (3) does not appear in many books but can be derived in a few steps using Parseval's relation for the ordinary Fourier transform, [5]. To the best of our knowledge the earliest notice of this relation is given by Rihaczek in [3, p.155].

The fractional Fourier transform generalizes these relations:

$$
\begin{array}{r}
\mathcal{A}_{r, s}(\tau \cos \phi, \tau \sin \phi)=\int_{-\infty}^{\infty} r_{a}\left(t_{a}\right) s_{a}^{*}\left(t_{a}-\tau\right) d t_{a} \\
\int\left|\mathcal{A}_{r, s}(\tau \cos \phi-u \sin \phi, \tau \sin \phi+u \cos \phi)\right|^{2} d u= \\
\int_{-\infty}^{\infty}\left|r_{a}\left(t_{a}\right)\right|^{2}\left|s_{a}\left(t_{a}-\tau\right)\right|^{2} d t_{a}
\end{array}
$$

The generalized relations reduce to their special cases when $\phi$ is taken as 0 or $\pi / 2$. The equation (4) states that a radial slice of the ambiguity function along the line $u=\tan (\phi) \tau$ can be calculated by cross-correlating the $a$ th domain fractional Fourier transforms of $r(t)$ and $s(t)$. Similarly the equation (5) can be interpreted as the projection of $\left|\mathcal{A}_{r, s}(\tau, u)\right|^{2}$ onto the line $u=\tan (\phi) \tau$. The proof of these relations follows from the rotation of the ambiguity function by the fractional Fourier transform, [5] (also see [10]).

In [5], we have studied a receiver for LFM signals making use of properties (4) and (5). The receiver operates in two stages. The first stage operates in the fractional domain which is orthogonal to the instantaneous frequency line of the chirp signal. The signal energy over ambiguity plane is projected to line (I). (The lines (I) and (II) are illustrated in Figure 2.) The second stage operates in the fractional domain which is aligned with the instantaneous frequency line as shown with line (II). A one-dimensional search operation along the lines (I) and (II) is conducted sequentially to detect the peak location in the ambiguity plane. In this paper, we present an optimality property for the first stage of this algorithm.

\section{DeteCtion OF SIGNALS WiTh UNKNOWN PARAMETERS}

We examine the following binary hypothesis test:

$$
\begin{array}{rlrl}
\mathrm{H}_{0} & : & & r(t)=w(t) \\
\mathrm{H}_{1} & : & r(t)=\sqrt{2 \mathrm{E}_{\mathrm{r}}} e^{j \phi} s(t ; \boldsymbol{\Theta})+w(t)
\end{array}
$$

We assume that $w(t)$ represents complex (circularly symmetric) Gaussian random process with zero mean and autocorrelation $r_{w}(t, u)=E\left\{w(t) w^{*}(u)\right\}=N_{0} \delta(t-u)$. The complex envelope signal $s(t)$ is normalized to unit energy, i.e. $\int_{0}^{T}|s(t)|^{2} d t=1$. $E_{r}$ denotes the received signal energy. The parameter $\phi$ represents the unknown carrier phase which is uniformly distributed in $[0,2 \pi]$. The $\boldsymbol{\Theta}$ parameter denotes the unknown delay and Doppler frequency of the transmitted waveform, $\Theta=[\tau u]$.

There are two different methodologies on detecting the presence or absence of the target, [18, p.420]:

1. GLRT Approach: In the GLRT approach, the unknown parameters are assumed to be non-random. The unknown parameters are estimated and then substituted in the hypothesis test. Following the substitution, the hypothesis test for known signal detection is applied, [18]. When the target is assumed to be slowly fluctuating as in [1, p.238] (when $E_{r}$ in (6) is taken as a Rayleigh random variable, i.e. Swerling-1 model) or assumed to be a non-fluctuating target at low SNR ( $E_{r}$ in (6) is non-random, but small) as in [2, p.255]; the decision statistics is given as follows:

$$
\begin{aligned}
\left|\int r(t) s^{*}(t ; \widehat{\boldsymbol{\Theta}}) d t\right|^{2} & =\left|\int r(t) s^{*}(t-\widehat{\tau}) e^{j 2 \pi t \widehat{u}} d t\right|^{2} \\
& =\left|A_{r s}(\widehat{\tau}, \widehat{u})\right|^{2} \gtrless \gamma
\end{aligned}
$$

To implement the GLRT detector, we need to form the estimates of $\tau$ and $u$. When both parameters are assumed to be non-random, the only feasible approach is the ML estimation. The ML estimator for $\Theta$ is given by [1, p. 276]:

$$
[\widehat{\tau}, \widehat{u}]=\arg \max _{\tau, u}\left|\mathcal{A}_{r s}(\tau, u)\right|^{2}
$$

The operation of the detector can be summarized as follows: First the location of peaks over the ambiguity plane is detected. Then the height of the ambiguity surface at the ML estimate (the peak level) is compared with a threshold. If threshold is exceeded, a target is declared at $(\widehat{\tau}, \widehat{u})$ parameters.

Bayesian Approach: Bayesian method assumes that there is a given a-priori probability distribution associated with the unknown parameters $\Theta,[18]$. The decision statistics is acquired by the marginalization of two hypotheses over unknown parameters:

$$
\begin{aligned}
& \frac{\int p\left(r(t) \mid \boldsymbol{\Theta}, \mathrm{H}_{1}\right) p_{\boldsymbol{\Theta} \mid \mathrm{H}_{1}}\left(\boldsymbol{\Theta} \mid \mathrm{H}_{1}\right) d \boldsymbol{\Theta}}{\int p\left(r(t) \mid \boldsymbol{\Theta}, \mathrm{H}_{0}\right) p_{\boldsymbol{\Theta} \mid \mathrm{H}_{0}}\left(\boldsymbol{\Theta} \mid \mathrm{H}_{0}\right) d \boldsymbol{\Theta}}=\ldots \\
& \ldots=\int \Lambda[r(t ; \boldsymbol{\Theta})] p_{\boldsymbol{\Theta} \mid \mathrm{H}_{1}}\left(\boldsymbol{\Theta} \mid \mathrm{H}_{1}\right) d \boldsymbol{\Theta}
\end{aligned}
$$

In the relation above, we note that $\mathrm{H}_{0}$ does not depend on $\Theta$. $\Lambda[r(t ; \boldsymbol{\Theta})]$ is the likelihood ratio of the hypothesis test in (6) for a fixed value $\Theta$. When the parameter $E_{r}$ of the hypothesis test is taken as a non-random variable, the likelihood ratio can be written as follows, [2, p.255]:

$$
\Lambda[r(t ; \boldsymbol{\Theta})]=e^{-\frac{E_{r}}{N_{0}}} I_{0}\left(\sqrt{2 E_{r}}\left|\mathcal{A}_{r, s}(\tau, u)\right| / N_{0}\right)
$$


Under low SNR conditions, which is the case of interest in general, $I_{0}(z)$ can be approximated as $I_{0}(z) \approx 1+z^{2}$ and the Neyman-Pearson test can be approximated as follows:

$$
\Lambda[r(t ; \boldsymbol{\Theta})] \approx\left|\mathcal{A}_{r, s}(\tau, u)\right|^{2} \gtrless \gamma
$$

Under these conditions, Bayesian receiver given in (9) reduces to

$$
\iint\left|\mathcal{A}_{r, s}(\tau, u)\right|^{2} p_{\tau, u}(\tau, u) d \tau d u \gtrless \gamma
$$

We would like to examine some special cases of the receiver given in (12). We follow the examples of Flandrin given in [13].

\section{Case 1: Known delay and Doppler frequency}

When both delay and Doppler frequency parameters are precisely known, a-priori probability distribution can be expressed as $p(\tau, u)=\delta\left(\tau-\tau_{a}\right) \delta\left(u-u_{a}\right)$. Under these conditions, Bayesian receiver reduces to the following:

$$
\iint\left|\mathcal{A}_{r s}(\tau, u)\right|^{2} p(\tau, u) d \tau d u=\left|\mathcal{A}_{r s}\left(\tau_{a}, u_{a}\right)\right|^{2} \gtrless \gamma
$$

The receiver is the optimal receiver for detecting presence or absence of the target at a point when the carrier phase is not unknown.

Case 2: Known delay but unknown Doppler frequency In this case, a-priori probability distribution for target parameters is $p(\tau, u)=\delta\left(\tau-\tau_{a}\right) p_{u}(u)$. Bayesian receiver for this case is $\int\left|\mathcal{A}_{r s}\left(\tau_{a}, u\right)\right|^{2} p_{u}(u) d u$. This relation can be expressed as follows:

$$
\begin{aligned}
\int\left|\mathcal{A}_{r s}\left(\tau_{a}, u\right)\right|^{2} p_{u}(u) d u=\cdots & \\
= & \int\left(\int r\left(t^{\prime}-\tau_{a} / 2\right) s^{*}\left(t^{\prime}+\tau_{a} / 2\right) e^{i 2 \pi u t^{\prime}} d t^{\prime}\right) \ldots \\
& \quad \ldots\left(\int r\left(t^{\prime \prime}-\tau_{a} / 2\right) s^{*}\left(t^{\prime \prime}+\tau_{a} / 2\right) e^{i 2 \pi u t^{\prime \prime}} d t^{\prime \prime}\right) p_{u}(u) d u \\
= & \iint r\left(t^{\prime}-\tau_{a} / 2\right) r^{*}\left(t^{\prime \prime}-\tau_{a} / 2\right) \ldots \\
\ldots & s^{*}\left(t^{\prime}+\tau_{a} / 2\right) s\left(t^{\prime \prime}+\tau_{a} / 2\right) \underbrace{\mathrm{E}_{u}\left\{e^{i 2 \pi u\left(t^{\prime}-t^{\prime \prime}\right)}\right\}}_{\Phi\left(t^{\prime}-t^{\prime \prime}\right)} d t^{\prime} d t^{\prime \prime}
\end{aligned}
$$

In the last equation $\Phi(\cdot)$ is the characteristic function of $p_{u}(u)$ and $E_{u}\{\cdot\}$ is the expected value operation with respect to the random variable $u$.

When $p_{u}(u)$ is uniformly distributed in $[-\mathrm{U} / 2, \mathrm{U} / 2]$, then $\Phi\left(t^{\prime}-t^{\prime \prime}\right)=\operatorname{sinc}\left(U\left(t^{\prime}-t^{\prime \prime}\right)\right)$. As [-U/2, U/2] interval expands to $[-\infty, \infty]$, that is as the uncertainty on the Doppler frequency increases, the characteristic function converges to $\delta\left(t^{\prime}-t^{\prime \prime}\right)$. Under this condition, the optimal receiver reduces to:

$$
\int\left|r\left(t-\tau_{a} / 2\right)\right|^{2}\left|s\left(t+\tau_{a} / 2\right)\right|^{2} d t \gtrless \gamma
$$

We see that the envelope correlator at a fixed delay $\tau_{a}$ is the decision statistics when the target delay is precisely known but Doppler frequency is an unknown.
When delay is not precisely known but distributed with $p_{\tau}(\tau)$ and Doppler parameter is interdependent from $\tau$, then the decision statistics becomes

$$
\int\left(\int|r(t-\tau / 2)|^{2}|s(t+\tau / 2)|^{2} d t\right) p_{\tau}(\tau) d \tau \gtrless \gamma
$$

The equation (16) can be interpreted as the expectation of equation (15) with respect to $\tau$ variable.

Case 3: Known Doppler frequency but unknown delay This case is the dual of the previous case. When similar steps are taken, we get the following decision statistics:

$$
\int\left|R\left(u-u_{a} / 2\right)\right|^{2}\left|S\left(u-u_{a} / 2\right)\right|^{2} d u \gtrless \gamma
$$

Here $R(\cdot)$ and $S(\cdot)$ is the ordinary Fourier transform of $r(t)$ and $s(t)$. The parameter $u_{a}$ denotes the value of the known Doppler frequency (actual Doppler).

\section{Case 4: Unknown delay and Doppler}

Assume that $p(\tau, u)=1 / A$ over region $A$ in the delay-Doppler plane with the area $A$, that is $p(\tau, u)$ is uniformly distributed over the region $A$. With this assumption, the optimal receiver becomes:

$$
\iint_{(\tau, u) \in \mathcal{A}} \frac{1}{A}\left|\mathcal{A}_{r s}\left(\tau_{a}, u\right)\right|^{2} d \tau d u \gtrless \gamma
$$

As the size of region $A$ gets larger, the decision statistics converges to

$$
\int_{-\infty}^{\infty} \int_{-\infty}^{\infty}\left|\mathcal{A}_{r s}\left(\tau_{a}, u\right)\right|^{2} d \tau d u=\int|r(t)|^{2} d t \int|s(t)|^{2} d t \gtrless \gamma
$$

Here the volume identity for ambiguity surface is used for the simplification, [4, p.209].

We note that the optimal detector in this case is the energy detector (radiometer). That is, when both range and Doppler parameters are assumed to be uniformly distributed in a large region of interest, the optimal Bayesian receiver is the energy detector.

Case 5: Unknown delay and Doppler over a sub-region. When the target is known to be in a region that is not vertically cut (Case 2) or horizontally cut (Case 3) in the ambiguity plane but diagonally oriented as shown in the Figure 1; the optimal receiver generalizing the previous cases can be written as follows:

$$
\int_{-\infty}^{\infty}\left|r_{a}\left(t_{a}\right)\right|^{2}\left|s_{a}\left(t_{a}-\tau\right)\right|^{2} d t_{a} \gtrless \gamma
$$

We note that Case 5 contains previous two cases. When the shaded area in Figure 1 is a vertical strip (known delay, unknown Doppler), the fractional domain parameter $a$ becomes zero and the receiver in (20) is equivalent to the one in (15). Similarly when the shaded area is a horizontal strip, the parameter $a$ is equal to 1 and the generalized receiver given in (20) is equivalent to (17). 


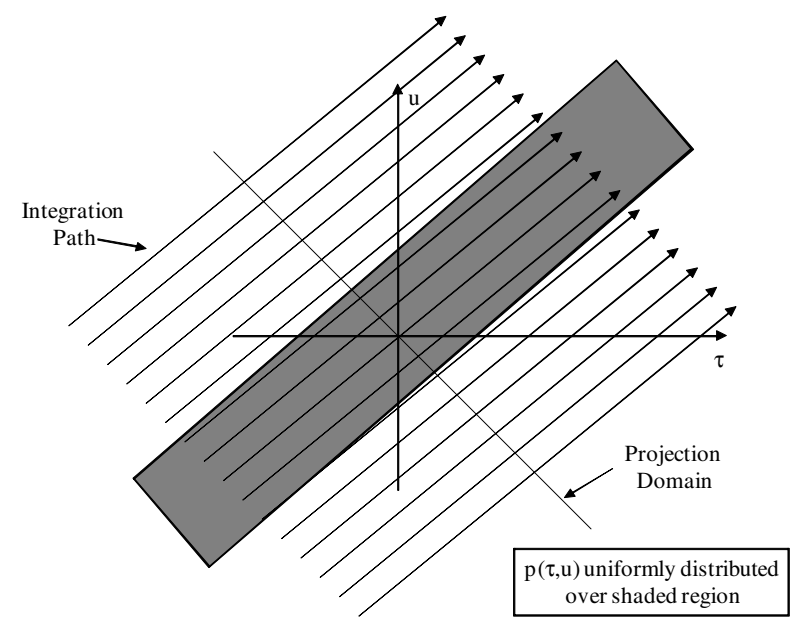

Fig. 1. Bayesian detector when $(\tau, u)$ uniformly is distributed over the shaded region.

\section{NUMERICAL COMPARISON}

We present the results of a simulation comparing the performance of different receivers. The simulation scenario is shown in Figure 2. We assume that a single target is known to be located on line (II). The target location is uniformly selected between the indicated points in Figure 2 as the closest and the furthest target location. We compare the performance of receivers making or not making use of this a-priori information. We use a chirp signal as the radar waveform. The numerical values for the parameters $d_{1}$ and $d_{2}$ which are shown in Figure 2 are given in Table I along with other signal and simulation parameters.

The first receiver assumes that the target location is precisely known and implements the matched filter receiver. This is equivalent to Case 1 of Section III. The processing gain of this receiver is $160(22 \mathrm{~dB})$ which is the code length under the discretization mentioned in Table I. The second receiver implements envelope correlation operation in the ath fractional domain. The third receiver is the conventional matched filter. The conventional matched filter evaluates $\mathcal{A}(\tau, 0)$ slice of the ambiguity function. The conventional matched filter can be considered to be matched to the zero Doppler frequency of the incoming signal. Since chirp signal is known to be Doppler tolerant, the processing gain of the system, in spite of the Doppler parameter mismatch, is expected to be significant. For the targets placed in the simulation, the processing gain of the conventional matched filter changes between 107 (20.2 $\mathrm{dB})$ and $122(20.9 \mathrm{~dB})$. The fourth receiver is the radiometer receiver. The radiometer calculates the total energy of the incoming signal in the time interval that an echo can reside. The time interval for the radiometer extends from the beginning of the echo from the closest target to the end of the echo from the furthest target.

The simulation results given in Figure 3 show that the envelope correlator in the fractional domains operates in close proximity to the optimal matched filter (receiver 1). This

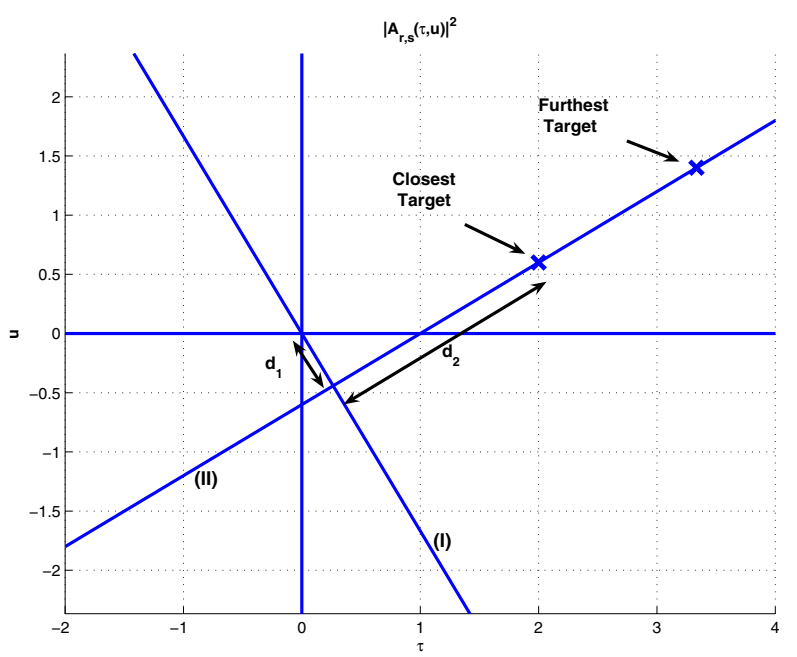

Fig. 2. Illustration of projection domains and targets

behaviour is expected since the energy content of chirp signals is localized along the line in the ambiguity plane containing the targets. The conventional matched filter suffers a loss of 3-4 dB in comparison to the optimal solution. The radiometer performs very poorly in comparison to the other detectors.

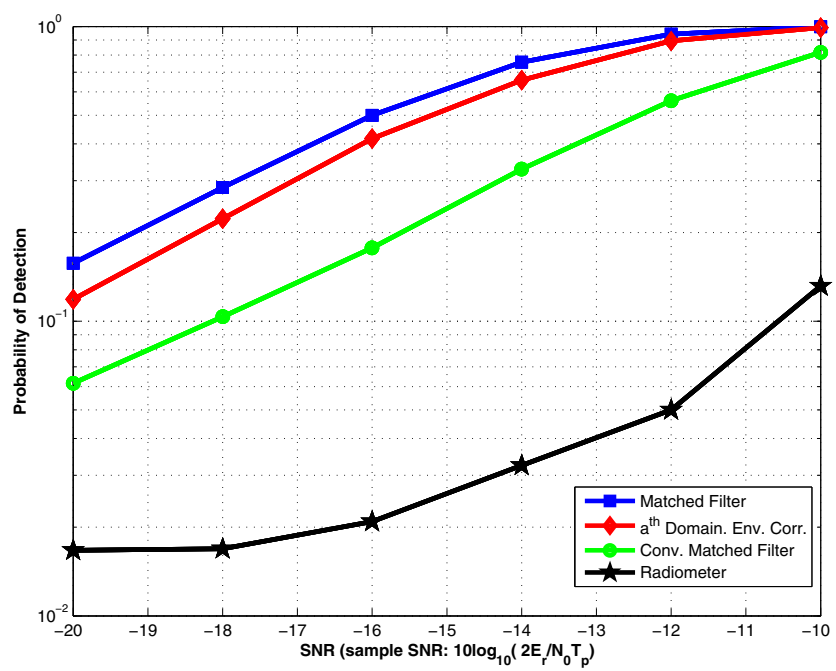

Fig. 3. Simulation results for $P_{F A}=0.01$.

\section{CONCLUSIONS}

In this paper, we present an optimality property of the detectors implementing projections over the ambiguity surface. We show that under low SNR conditions, non-fluctuating targets known to be lying along the straight lines in the ambiguity plane can be optimally detected in the Bayesian setting. This result shows the optimality of the first stage of the algorithm proposed earlier in [5].

\section{ACKNOWLEDGMENT}

Author would like to acknowledge the support of TUBITAK for this work under grant no. 106E187. 
TABLE I

SiMULATION PARAMETERS

\begin{tabular}{ll} 
Signal Parameters & \\
\hline Pulse width $\left(T_{p}\right)$ & $: 8 \mathrm{sec}$ \\
Chirp rate $(k)$ & $: 1.5 \mathrm{~Hz} / \mathrm{sec}$ \\
$d_{1}$ & $: 0.5$ \\
minimum value for $d_{2}$ & $: 3$ \\
maximum value for $d_{2}$ & $: 5$ \\
codelength (processing gain) & $: 160$ \\
& \\
Fractional Fourier Transform Calculation & $:[-10,10] \mathrm{secs}$ \\
\hline input signal span & $: 1 / 20 \mathrm{secs}$ \\
sampling period & $: \mathrm{x} 20 / 12$ \\
signal oversampling & $: 1.62$ \\
projection domain & \\
& \\
Other Parameters & $: 0.01$ \\
\hline False Alarm Probability & $: 5000$
\end{tabular}

\section{REFERENCES}

[1] H. L. V. Trees, Detection, Estimation and Modulation Theory, Part III. New York: Wiley, 1968.

[2] R. N. McDonough and A. D. Whalen, Detection Of Signals In Noise. London, CA: Academic Press, 1995

[3] A. W. Rihaczek, Principles of High Resolution Radar. New York: McGraw-Hill, 1969.

[4] R. E. Blahut, Theory of Remote Image Formation. Cambridge, England: Cambridge University Press, 2004.

[5] C. Candan, "On the Implementation of Optimal Receivers For LFM Signals Using Fractional Fourier Transform," IEEE Radar Conference, 2008.

[6] M. Wang, A. K. Chan, and C. K. Chui, "Linear Frequency-modulated Signal Detection Using Radon-Ambiguity Transform," IEEE Trans. Signal Processing, vol. 46, no. 3, pp. 571-586, 1998.

[7] A. K. Ozdemir and O. Arikan, "Fast Computation of the Ambiguity Function and the Wigner Distribution on Arbitrary Line Segments,' IEEE Trans. Signal Processing, vol. 49, pp. 381-393, February 2001.

[8] O. Akay and G. F. Boudreaux-Bartels, "Fractional convolution and correlation via operator methods and an application to detection of linear FM signals," IEEE Trans. Signal Processing, vol. 49, no. 5, pp. 979-993, 2001.

[9] A. K. Ozdemir, Novel Time-Frequency Analysis Techniques for Deterministic Signals. PhD thesis, Bilkent University, Ankara, Turkey, 2003.

[10] O. Arikan and A. K. Ozdemir, "System and Method for Detection and Tracking of Targets," United States Patent 6636174, May 2007.

[11] F. Hlawatsch and G. F. Boudreaux-Bartels, "Linear and quadratic timefrequency signal representations," IEEE Signal Processing Magazine, vol. 9, no. 2, pp. 21-67, 1992

[12] G. Maltz and F. Hlawatsch, "Wigner Distributions (nearly) everywhere: time-frequency analysis of signals, systems, random processes, signal spaces and frames," Elsevier Signal Processing, vol. 83, pp. 1355-1378, 2003.

[13] P. Flandrin, "On Detection-Estimation Procedures In The TimeFrequency Plane," Proc. IEEE Int. Conf. Acoust. Speech Signal Process., pp. 2331-2334, 1986.

[14] P. Flandrin, "A Time-Frequency Formulation Of Optimal Detection," IEEE Trans. Signal Processing, vol. 36, no. 9, pp. 1377-1384, 1988

[15] A. M. Sayeed and D. L. Jones, "Optimal Detection Using Bilinear Time-Frequency and Time-Scaler Representations," IEEE Trans. Signal Processing, vol. 43, no. 12, pp. 2872-2883, 1995.

[16] A. M. Sayeed and D. L. Jones, "Corrections to Optimal Detection Using Bilinear Time-Frequency and Time-Scaler Representations," IEEE Trans. Signal Processing, vol. 45, no. 3, pp. 761-762, 1997.

[17] H. M. Ozaktas, The Fractional Fourier Transform with Applications in Optics and Signal Processing. England: John Wiley and Sons, 2001.

[18] S. M. Kay, Fundamentals of Statistical Signal Processing, Volume 2 . Detection Theory . Prentice Hall, 1998. 